FORMATLON Formation emploi

Revue française de sciences sociales

144 | Octobre-Décembre

Le décrochage scolaire à l'aune des inégalités

territoriales: nouvelles perspectives?

\title{
Présentation de l'ouvrage de A. Dupray et \\ E. Quenson, Sept ans de vie professionnelle des jeunes: entre opportunités et contraintes
}

Stéphane Moulin

\section{OpenEdition}

\section{Journals}

Édition électronique

URL : http://journals.openedition.org/formationemploi/6781

DOI : 10.4000/formationemploi.6781

ISSN : 2107-0946

Éditeur

La Documentation française

Édition imprimée

Date de publication : 31 décembre 2018

Pagination : 177-180

ISSN : 0759-6340

Référence électronique

Stéphane Moulin, «Présentation de l'ouvrage de A. Dupray et E. Quenson, Sept ans de vie

professionnelle des jeunes : entre opportunités et contraintes », Formation emploi [En ligne], 144 | OctobreDécembre, mis en ligne le 31 décembre 2018, consulté le 06 janvier 2021. URL : http://

journals.openedition.org/formationemploi/6781; DOI : https://doi.org/10.4000/formationemploi.6781

(c) Tous droits réservés 


\title{
Note de Lecture
}

\section{Présentation de l'ouvrage de A. Dupray et E. Quenson, Sept ans de vie professionnelle des jeunes: entre opportunités et contraintes}

\author{
Par Stéphane Moulin \\ Professeur agrégé, Département de sociologie, université de Montréal, Canada
}

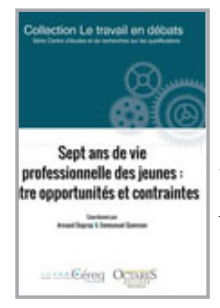

Comment les jeunes se sont-ils insérés sur le marché du travail, en France, pendant les années 2004 à 2011, marquées par la crise économique de 2008 ? Telle est la question centrale de l'ouvrage "Sept ans de vie professionnelle des jeunes : entre opportunités et contraintes". Pour y répondre, Arnaud Dupray et Emmanuel Quenson ont réuni un collectif de vingt chercheurs, économistes et sociologues, ayant analysé les données de l'enquête Génération 2004 du Céreq. Les enquêtes Génération présentent l'originalité d'interroger et de suivre l'ensemble des sortants d'une même année du système éducatif. Comme les générations sont construites en fonction de la date de sortie de la formation initiale et non de l'année de naissance, cela permet de s'assurer que tous les jeunes interrogés sont entrés sur le marché du travail dans la même conjoncture. Cela en fait donc l'enquête idéale à la fois pour analyser l'insertion et pour apprécier l'impact d'une crise économique.

Trois contributions évaluent directement l'impact de la crise économique, en comparant l'insertion des jeunes sortants en 1998, qui n'ont pas connu la crise, avec celle des sortants de l'année 2004, sur les sept premières années de leur vie active. Ces analyses confirment que la crise s'est traduite par une dégradation de l'insertion des jeunes sortants qui ont connu des épisodes de chômage plus nombreux et plus longs (Calmant \& Ménard), ont été plus souvent contraints à travailler à temps partiel (Couprie \& Joutard) et ont moins souvent accédé aux emplois de cadres (Di Paola \& Moullet). Si ces résultats ne surprennent pas, chacune de ces analyses utilise des techniques avancées de statistique pour les démontrer. Les contributions nuancent aussi finement la portée de cette dégradation qui varie selon le niveau de diplôme ou l'origine sociale. On apprend ainsi, par exemple, que ce sont surtout les jeunes peu ou faiblement diplômés qui ont été davantage touchés par les risques de chômage ou de temps partiel involontaire. Il ressort 
aussi que les jeunes avec le moins de réseaux professionnels (dont aucun des deux parents n'était cadre) ont moins accédé aux emplois de cadres. C'est là sans doute que réside l'intérêt principal de la comparaison proposée entre les deux générations. En effet, les contributions montrent ainsi selon quelles modalités les jeunes ne sont pas tous égaux face à la crise : en période de ralentissement économique, le diplôme protège encore davantage contre le chômage ou les contraintes temporelles subies et le rôle de la socialisation familiale dans l'accès aux emplois de cadre est accentué.

Toutes les contributions de l'ouvrage exploitent le caractère longitudinal de l'enquête qui permet de suivre les jeunes pendant sept ans en les réinterrogeant à trois reprises en 2007, 2009 et 2011. Certains chercheurs mettent d'ailleurs au centre de leur interrogation les changements qui s'opèrent pendant le processus d'insertion. Ils confirment l'intérêt d'aborder l'insertion dans un plus long terme, ici sept ans, dans la mesure où les résultats des analyses diffèrent parfois significativement entre le court et le long terme. Ilardi \& Sulzer montrent ainsi que l'apprentissage favorise la stabilisation dans l'emploi à court-terme, mais pas à moyen-terme. Béduwé \& Dupray dégagent d'importants décalages entre appréciation salariale et niveau de salaire. Ils analysent en outre ce qui conduit les jeunes à modifier le jugement qu'ils portent sur leurs salaires. Enfin, Mora analyse les reprises d'études qui, jusqu'à récemment, étaient relativement moins étudiées dans le contexte français, marqué par un modèle d'insertion séquentiel des études vers le travail. Si la fréquence des retours aux études a doublé de la génération des sortants de 1998 à celle des sortants de 2004, elle montre que la crise n'a pourtant pas provoqué de sursaut visible des retours en formation et que cette croissance s'inscrit plutôt dans une tendance de long terme. Si la crise ne semble pas amplifier le phénomène, les jeunes ne profitent pas tous, pour autant, des retours en formation : l'obtention de diplômes après la sortie de la formation initiale n'améliore les situations professionnelles que lorsqu'ils sont d'un niveau supérieur au bac.

Quelques contributions ré-abordent des facettes spécifiques du processus d'insertion en contexte de crise. Elles offrent ainsi également des contributions théoriques originales. Analysant les canaux qui permettent aux jeunes de trouver des emplois, De Larquier, Rieucau \& Brinbaum opèrent une distinction entre trois différents segments du marché du travail (externe, organisé et informel) et éclairent ainsi les trajectoires de stabilisation professionnelle. Jaoul Grammare \& Lemistre discutent de la mesure du déclassement et montrent que les résultats diffèrent significativement selon que l'on opte pour une approche normative du phénomène (dite objective) ou pour une mesure subjective selon le diplôme ou selon les compétences. Ils invitent à se méfier des mesures subjectives qui sont liées à des priorités individuelles et peuvent traduire une certaine forme de résignation. Enfin, Brinbaum \& Issehnane discutent des indicateurs de qualité de l'emploi et proposent une approche factorielle qui prend en compte les différentes caractéristiques des emplois occupés par les jeunes. Elles montrent que si le désavantage des jeunes d'origine maghrébine s'efface en 2007, il réapparaît en 2011, ce qui témoigne de l'effet discriminant 
de la crise selon l'origine. C'est donc là une autre dimension des effets inégaux de la crise qui cantonne les jeunes d'origine maghrébine dans les emplois de moins bonne qualité.

Enfin, deux contributions adoptent le prisme du genre comme entrée principale pour analyser les modalités actuelles de l'insertion professionnelle. De fait, il est d'autant plus essentiel d'adopter cette lecture genrée que dans les dernières décennies, la proportion des femmes diplômées de l'enseignement supérieur a cru beaucoup plus vite que celle des hommes, dans un double contexte de ségrégation genrée des formations et de discrimination des femmes dans l'accès aux catégories de cadres. Ces transformations contradictoires manifestent sans doute une des tensions sociales les plus importantes dans l'emploi. Di Paola \& Moullet montrent qu'en dépit d'une législation renforcée en matière d'égalité hommes-femmes, les femmes accèdent toujours plus difficilement et plus tardivement à la catégorie de cadre. Couppié \& Épiphane révèlent que les femmes issues des formations scientifiques subissent non seulement une pénalité salariale dans les premières années de vie active, mais aussi que cette pénalité s'accroît avec le temps, notamment parce que les hommes, à l'inverse des femmes, connaissent une chance accrue d'accéder aux fonctions d'encadrement.

On pourra regretter quelques manques, comme l'analyse des situations et des stratégies des jeunes au chômage, tant d'un point de vue longitudinal qu'en comparaison avec l'enquête Génération 1998. On peut se demander si les conditions d'inégalités mises en lumière dans ce volume sont les prémices d'évolutions plus systémiques ou de redistribution des inégalités à l'aune du cycle de vie, à l'image des transformations mises en évidence pour le Canada (Moulin, 2017). D'ailleurs, un chapitre comparatif international, ne serait-ce que de cadrage, aurait trouvé sa place, en dégageant par exemple d'éventuelles spécificités de la situation française relativement à celle d'autres pays de l'OCDE (Organisation de coopération et de développement économiques). Toutefois, on sait aussi que des examens comparatifs plus ambitieux se heurtent à la disponibilité de données qui soient comparables et à l'usage de concepts et catégories dont les significations peuvent varier d'un pays à l'autre (Moulin, 2010).

Ces quelques limites n’enlèvent rien à la qualité des analyses proposées. Elles ont le mérite de pointer les différences entre des épreuves qui seraient de court-terme et liées à la dégradation brutale du cycle économique sur la période 2008-2009 et des évolutions davantage inscrites dans des tendances de long terme, comme la montée des reprises d'études et de formation pour des jeunes déjà entrés à plein temps sur le marché du travail. Cette dernière tendance peut d'ailleurs amener à interroger la vision traditionnelle du cloisonnement, en France, entre le temps de l'éducation et de la formation et le temps de l'emploi (Van de Velde, 2010). Les contributions proposées ont aussi le mérite de réaffirmer que les jeunes sont loin d'être tous égaux face à la crise : ce sont les moins diplômés, les jeunes issus des milieux professionnels les plus modestes, les femmes et les immigrés d'origine maghrébine qui demeurent les plus touchés par la dégradation des difficultés d'insertion. 


\section{Référence de l'ouvrage}

Dupray A., Quenson E. (coord.) (2018), Sept ans de vie professionnelle des jeunes : entre opportunités et contraintes, Ouvrage en co-édition n ${ }^{\circ} \mathrm{HC}$, Céreq/Octarès, collection « Le travail en débats », juillet, 204 p.

\section{Bibliographie}

Moulin S. (2017), « Une nouvelle génération d'inégalités dans les parcours de vie? Tensions sociales et genrées ", Revue Jeunes et Société, Vol. 2(2) pp. 23-49. http:// rjs.inrs.ca/index.php/rjs/article/view/109

Moulin S. (2010), "Statistical categorization of young people's entry into the labour market: a France/Canada comparison", International Journal of Comparative Sociology, Vol. 51(1-2), pp. 85-110.

Van de Velde C. (2008), Devenir adulte : sociologie comparée de la jeunesse en Europe, Paris, Presses universitaires de France. 